\title{
Feature Extraction of Early AE Signal Based on the Lifting Wavelet and
}

\section{Empirical Mode Decomposition}

\author{
Xuejun $\mathrm{Li}^{\mathrm{a}}{ }^{\text {, Zongqun Deng }}{ }^{\mathrm{b}}$, Lingli Jiang ${ }^{\mathrm{c}}$, Kuanfang $\mathrm{He}^{\mathrm{d}}$ \\ ( Hunan Provincial Key Laboratory of Health Maintenance for Mechanical Equipment, Hunan Uni- \\ versity of Science and Technology, Xiangtan 411201, Hunan, China.) \\ ahnkjdxlxj@163.com; bdengzongqun@163.com; 'linlyjiang@163.com; 'hkf791113@163.com
}

Key words: lifting wavelet; EMD; envelope analysis; feature extraction

\begin{abstract}
To solve the problem of difficult to extract fault features from the early weak AE signal, a method combining lifting wavelet with EMD is proposed. Firstly, the lifting wavelet is used to de-noise the AE signal, then the signal after de-noising is decomposed by EMD, and gets all kinds of different frequency IMFS. Finally, the most relevant IMF with the original signal depends on the size of the correlation coefficients, and the fault characteristic signal is extracted via combining time- frequency analysis and envelope spectrum analysis. The result of simulation and test signal shows that this method can effectively extracts the failure characteristics from early AE signal.
\end{abstract}

\section{Introduction}

Acoustic Emission (AE) technology is a technique that uses of snapshot flexibility and responsiveness set the wave phenomenon, which is produced by material (including the atoms, molecules and particle swarm) to identify and understand the material and structure of the internal state. The technology has some advantages in the early mechanical failure diagnosis. Such as anti-interference ability and obvious failure features. The traditional wavelet analysis is a good time-frequency analysis. As the frequency of transformation, there is slow speed of operation and other issues.

The lifting wavelet is also called the second generation wavelet, and the transformation of this wavelet uses the lifting algorithm, which does not rely on the Fourier transformation, and the operating speed of the lifting wavelet is quick, and easy to act according to signal's characteristic structure wavelet primary function. Therefore, the lifting wavelet obtained the widespread application, especially in the signal processing and the failure diagnosis [1-4]. The Empirical Model Decomposition (EMD) is a method, which has the auto-adapted characteristic and non-steady signal analysis, at the same time this method can put the complex seismic signal adaptive disintegrated into the sum of some intrinsic mode function (IMF)[5-7]. But if the actual sound transmitting message characteristic signal is weak, the noise of the fault background will be strong etc, so in the EMD decomposition process may have the modality aliasing phenomenon, and may influence the accuracy and the validity of the analysis results. Therefore, Beijing University of Technology of Cui Lingli[8] etc proposed a demodulation method combining the second generation wavelet and EMD, which can accurately extract high frequency modulation fault features. Wang Fengli[[9] etc combines lifting wavelet and local wave effectively to extract fault feature form rotor rubbing, which gets good results. But the research method only for vibration signal, and the early failure of the AE signal has not been studied with the method.

In this paper, the method of combining lifting wavelet, EMD, correlation analysis, spectrum analysis and envelope analysis method was proposed, which was used in AE early fault signal feature extraction, achieved good results and showed the accuracy and practical of this method.

\section{Principles of Lifting Wavelet Transformation}

Lifting scheme is proposed in 1995 by the Sweldens[10], The wavelet function is no longer generated by the translation and scaling one function, and all operations are carried out in the time domain. Not only for signal separation in various frequency bands, but also can be designed with specific features of the wavelet. Lifting wavelet transform process is divided into 3 phases[11]: Split, Predict, Update. 
(1)Split. Samples of the input data $\{s(k), k \in z\}$ are divided into odd sample sequence $s_{0}(k)$ and even samples sequence $s_{e}(k)$

$$
\begin{aligned}
& s_{0}(k)=s(2 k+1) \quad k \in z \\
& s_{e}(k)=s(2 k) \quad k \in z
\end{aligned}
$$

(2)Predict. Predictor coefficient set $P(\cdot)$, and use the predicted value $P\left[s_{e}(k)\right]$ of the Even sample $s_{e}(k)$ to predict (or interpolation) odd samples $s_{0}(k)$, they will have residual signal by subtracting the true and predicted value, and define the residual signal as the detail signal

$$
d(k)=s_{0}(k)-P\left[s_{e}(k)\right] \quad k \in z
$$

(3)Update. $U(\cdot)$ is set to updater coefficient. To make some global properties of the original signal set remain in their $\operatorname{subset}_{s_{e}}(k)$, on the basis of the detail signal $d(k)$ to update $s_{e}(k)$, and the result is defined as the approximation signal

$$
c(k)=s_{e}(k)+U[d(k)] \quad k \in z
$$

The reconstruction of the lifting wavelet is the reverse process of the decomposition process, the reconstruction process is as follows:

$$
\begin{array}{ll}
s_{0}(k)=d(k)-P\left[s_{e}(k)\right] & k \in z \\
s_{e}(k)=c(k)-U[d(k)] & k \in z
\end{array}
$$

\section{Empirical Mode Decomposition and Envelope Spectrum Analysis}

A complex signal can be decomposed into sum of several intrinsic mode function (IMF) by EMD method, the decomposition step are as follows [12]:

(1) Determine all local minimums of the signal, then use cubic spline to connect all local maximum points and minimum points, formulate the upper and lower envelope line which should contain all of the data points.

(2) Calculated out the average of the upper and lower envelope and denoted as $m_{1}$, calculated as follows:

$$
x(t)-m_{1}=h_{1}
$$

Ideally, if $h_{1}$ is a IMF, then it is the first IMF component of $x(t)$.

(3) If $h_{1}$ does not meet the IMF conditions, regard $h_{1}$ as initial data, repeat steps (1) and (2) to get the average $m_{11}$ of the upper and lower envelope, then determine whether $h_{11}=h_{1}-m_{11}$ meet the conditions of IMF, if not then repeat it $k$ times, obtain $h_{1(k-1)}-m_{1 k}=h_{1 k}$ and made $h_{1 k}$ to meets the conditions of IMF. Denote $c_{1}=h_{1 k}$, then it is the first IMF component of $x(t)$.

(4) Separated $c_{1}$ from $x(t)$, obtained that:

$$
r_{1}=x(t)-c_{1}
$$

Regard $r_{1}$ as initial data and repeat (1)-(3), gained the second IMF component $c_{2}$ of $x(t)$, repeat it $n$ times and obtained $n$ IMF components of $x(t)$. Then

$$
x(t)=\sum_{i=1}^{n} c_{i}+r_{n}
$$

Where $r_{n}$ in the expression is the residue function, which represents the average trend of signals.

The each intrinsic mode function $c_{i}$ of type (9) with Hilbert transformation gets

$$
\hat{c}_{i}(t)=\frac{1}{\pi} \int_{-\infty}^{\infty} \frac{c_{i}(\tau)}{t-\tau} d \tau
$$

So can construct analytical signal

$$
z_{i}(t)=c_{i}(t)+j \hat{c}_{i}(t)=a_{i}(t) e^{j \phi_{i}(t)}
$$


By type (11) can get amplitude function

$$
a_{i}(t)=\sqrt{c_{i}^{2}(t)+\hat{c}_{i}^{2}(t)}
$$

And $a_{i}(t)$ is the envelope signal of original signal, and obtains the envelope spectrum of the original signal by spectrum analysis.

\section{Method of Fault Feature Extraction}

The method of EMD is a very kind of non-stationary signal processing method with adaptive, it can do adaptive time-frequency decomposition according to signal local time-varying characteristic, and can get high time-frequency resolution. Therefore, the method that combining lifting wavelet, EMD, correlation analysis, spectrum analysis and envelopment analysis is proposed in this text, the impact of noise for the decomposition on the EMD is overcame, the quality of EMD decomposition is improved, the generation of false modal components in the process of EMD decomposition is avoided, so the early fault feature of acoustic emission single can be extracted quickly and accurately, the procedures as follows:

(1) The early fault feature of acoustic emission is done de-noising processing using for lifting wavelet;

(2) The single of acoustic emission after de-noising processing is done EMD composition, and get intrinsic mode functions of all kinds' different frequency;

(3) Make sure the correlation of each intrinsic mode functions and original signal using relevant function;

(4) The intrinsic mode functions belong to the bigger correlation is done to spectrum analysis and envelopment analysis, and extract information of fault feature.

\section{Simulation Experiments}

Actually, all original process of acoustic emission are gusty, typical AE signal produced by mechanical failure presents fast trend in raise stage, presents exponential decay oscillation in decline stage, whose envelope curve is triangular shape[13]. Therefore, according to the AE simulation signal model in references [14], acoustic emission signal is simulated via three exponential decay signals. The expression is as follows:

$$
\begin{gathered}
x(t)=2\left[\exp \left(-2 \pi\left(\left(t-t_{1}\right) / \beta_{1}\right)^{2}\right)\right] \times \sin \left(2 \pi f_{1}\left(t-t_{1}\right)\right)+2\left[\exp \left(-2 \pi\left(\left(t-t_{2}\right) / \beta_{2}\right)^{2}\right)\right] \times \\
\sin \left(2 \pi f_{2}\left(t-t_{2}\right)\right)+2\left[\exp \left(-2 \pi\left(\left(t-t_{3}\right) / \beta_{3}\right)^{2}\right)\right] \times \sin \left(2 \pi f_{3}\left(t-t_{3}\right)\right)
\end{gathered}
$$

This simulation signal is composed of three pulse signal, centered by time $t_{1}, t_{2}, t_{3}, f_{1}, f_{2}, f_{3}$ are frequency of three harmonic signal, each parameter are as follows: $f_{1}=60 \mathrm{kHz}, f_{2}=70 \mathrm{kHz}, f_{2}=70 \mathrm{kHz}, f_{3}=80 \mathrm{kHz}, t_{1}=0.8 \mathrm{~ms}, t_{2}=0.9 \mathrm{~ms}, t_{3}=1.0 \mathrm{~ms}$, $\beta_{1}=0.0001, \beta_{2}=0.00015, \beta_{3}=0.0002$, sampling frequency is $500 \mathrm{kHz}$, the simulation signal is shown in Fig. 1.

The acoustic emission signal with Gaussian white noise is shown in Fig. 2, AE signal in early weak fault is simulated. It can be seen from the figure that the SNR (signal to noise ratio) of AE signal in early weak fault is low. Useful fault information is submerged in a lot of background noise. So, the key to get useful fault characteristic signal is to make a good noise canceling for the collected AE signal. In order to get fault characteristic signal, spectrum analysis is used. The lifting wavelet of AE signals without noise is shown in Fig. 3, and the power spectrum of de-noising signal is shown in Fig. 4. According to many experiments in MATLAB, the 2-level db3 wavelet decomposition has a good effect.

The Fig. 4 shows that the lifting wavelet has some noise reduction effect in early weak AE fault signal. But, the de-noised signal spectrum in addition to $60 \mathrm{kHz}, 70 \mathrm{kHz}, 80 \mathrm{kHz}$ pulse signal, there are still some relatively large amplitude of less than $60 \mathrm{kHz}$ the noise signal, and the effect of noise reduction is not very good. 


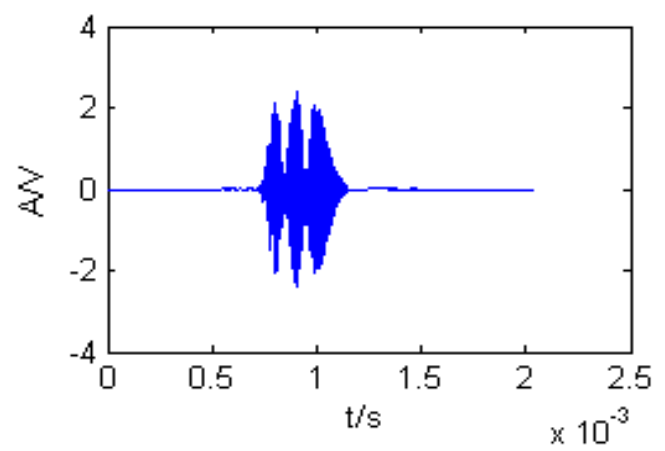

Fig. 1. AE simulation signals

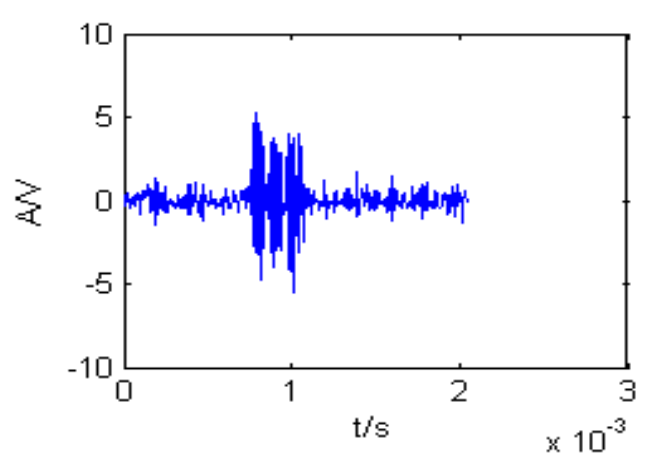

Fig. 3. The AE simulation signals without noise

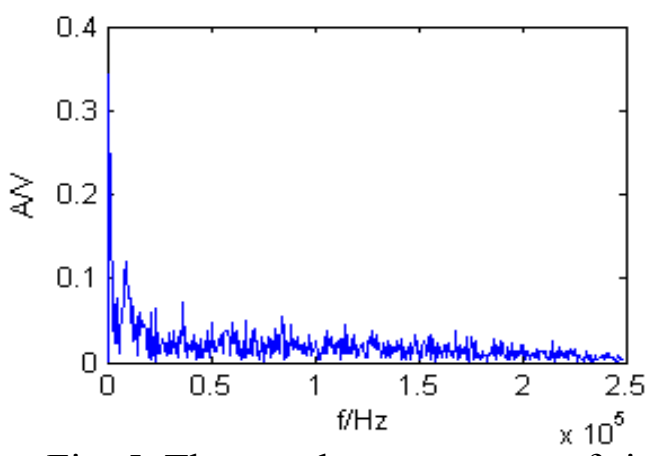

Fig. 5. The envelope spectrum of signal before de-noising

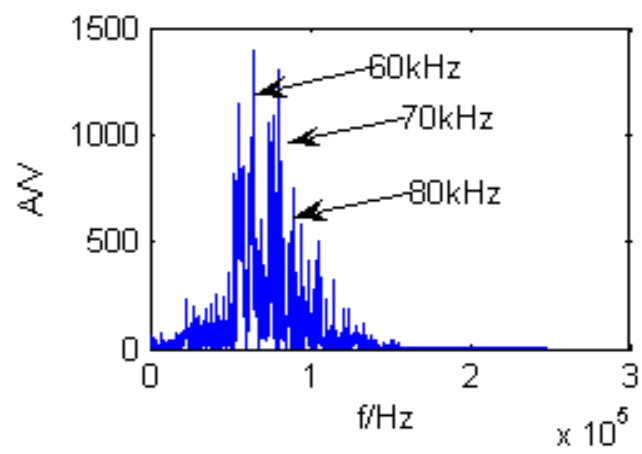

Fig. 7. The spectrum chart of IMF2 before de-noising

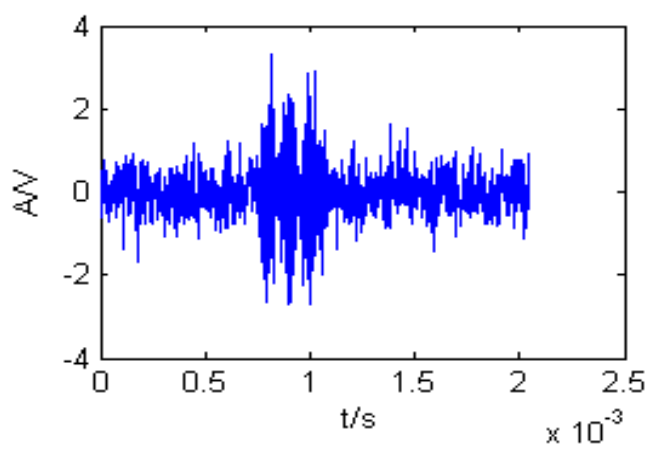

Fig. 2. AE simulation signals with noise

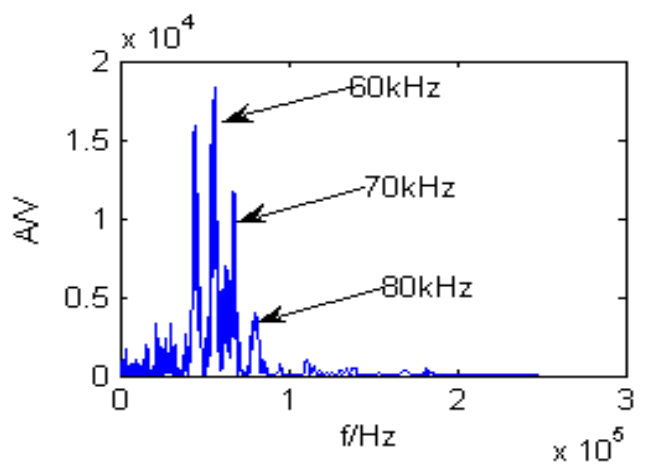

Fig. 4. Power spectrum of de-noising signal

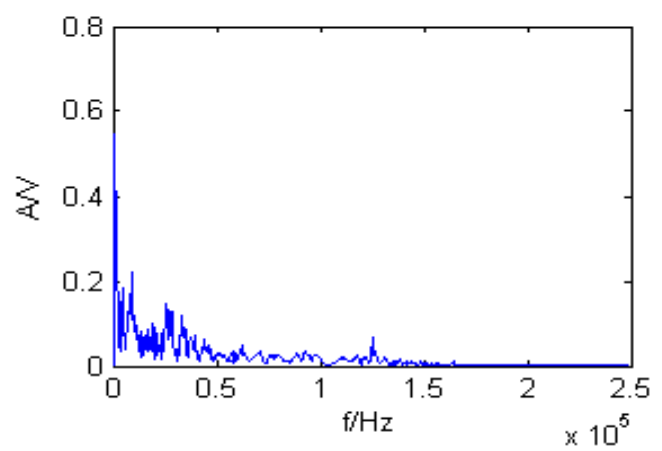

Fig. 6. The envelope spectrum of signal after de-noising

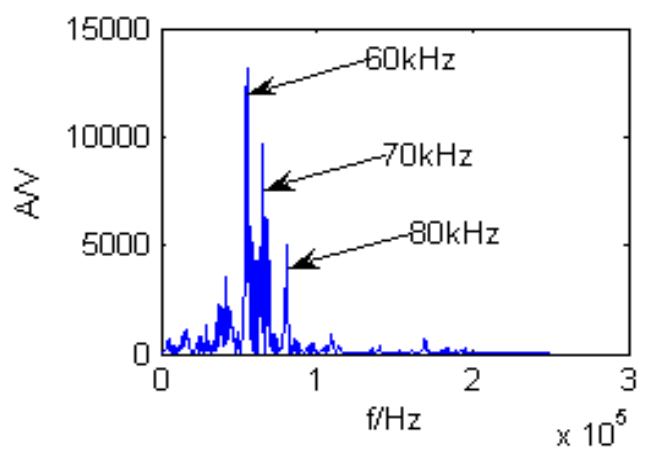

Fig. 8. The spectrum chart of IMF1 after de-noising

Fig. 5 and Fig. 6 respectively is the envelope spectrum of signal before and after de-noising. Through the Fig. 5 and Fig. 6 compared with that by lifting wavelet de-noising, high frequency part is removed, frequency components become less, and leave only useful part, which are helpful for extracting signal fault characteristic information. 
In order to accurately extract fault features, using EMD to decompose the signal after the noise reduction, and using cross-correlation function to determine the most relevant intrinsic mode function (IMF) with the original signal, then spectral analysis. Fig. 7 and Fig. 8 respectively is the spectrum chart of the second IMF before signal de-noising and the first IMF after signal de-noising.

A combination of the simulation result, if the signal noise content of large, then EMD decomposition can produce false component, and impact the quality of EMD decomposing. But the combination of the lifting wavelet and EMD, which can overcome the deficiencies of EMD, and accurately extract the weak signal fault characteristic from a lot of background noise.

\section{The Analysis of Experimental Results}

On American comprehensive machinery fault simulator, the bearing fault AE signal of outer ring is collected with AE testing equipment. The sampling frequency sets to $500 \mathrm{kHz}$ in experimental process. Fig. 9 is the AE signal time chart of experimental bearing, and the original signal contains plenty of noise signals and fails to show valuable acoustic emission signal information. Fig. 10 is AE signal after de-noising through lifting wavelet. The $12 \mathrm{IMF}$ and a residual function $r$ can be got with the de-noising AE signal after EMD. Fig. 11 is time-frequency diagrams of the first 8 IMF components. Table 1 is correlation coefficient among $8 \mathrm{IMF}$ with signal before and after de-noising, and $\mathrm{S}$ is the original signal, $\mathrm{S} 1$ is the signal after lifting wavelet de-noising. From the table can get conclusion that the first two IMFS have bigger correlation with signal after de-noising. The sixth and seventh IMF has bigger correlation with signal before de-noising.

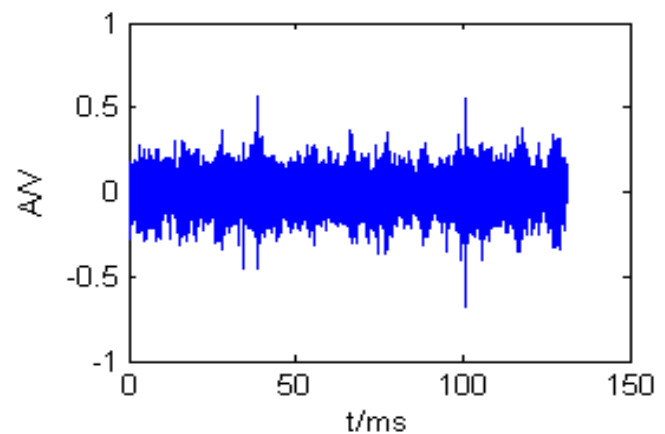

Fig. 9. The AE signal of bearing fault
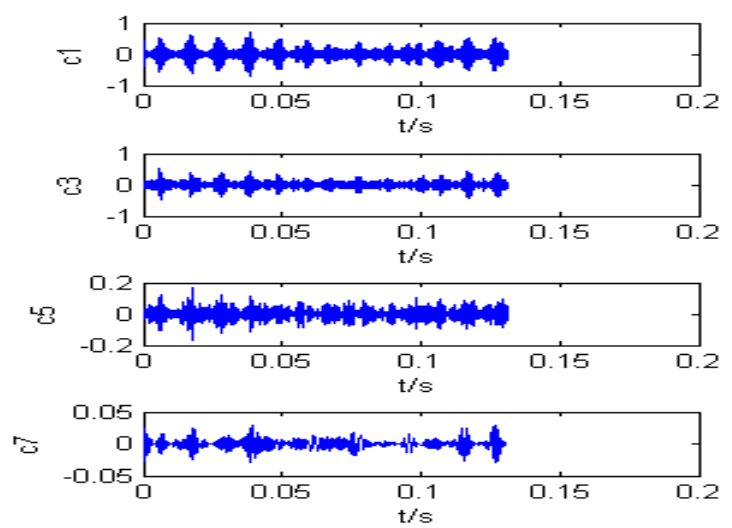

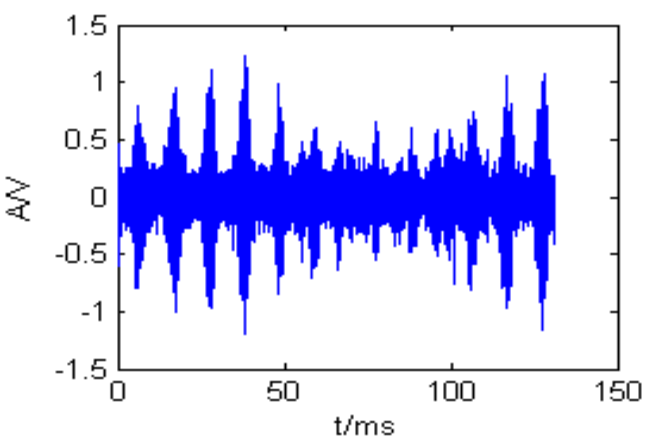

Fig. 10. The AE signal after de-noising
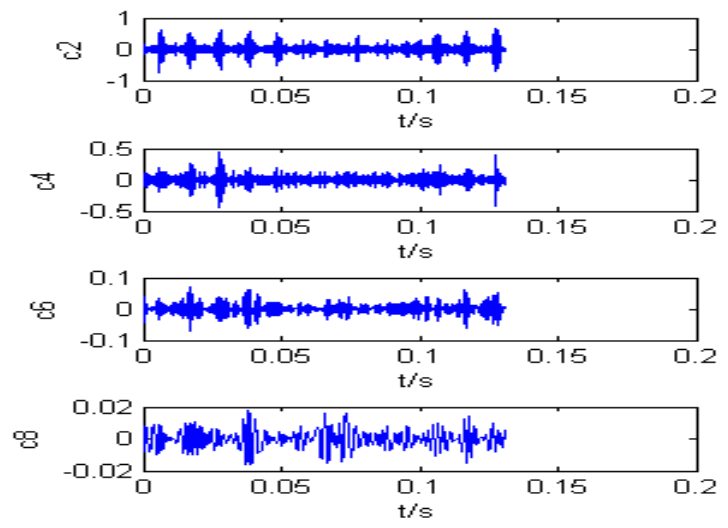

Fig. 11. Time-domain chart of the first 8 IMF

Table 1 The IMF correlation coefficient size of before and after and signal de-noising

\begin{tabular}{ccccccccc}
\hline & IMF1 & IMF 2 & IMF 3 & IMF 4 & IMF 5 & IMF 6 & IMF 7 & IMF 8 \\
\hline S & $\mathbf{0 . 1 6 7 4 8}$ & $\mathbf{0 . 0 0 7 1 1}$ & $\mathbf{0 . 0 0 2 8 1}$ & $\mathbf{0 . 0 3 7 7 8}$ & $\mathbf{0 . 1 4 6 4 6}$ & $\mathbf{0 . 3 1 2 6 6}$ & $\mathbf{0 . 2 2 1 7 6}$ & $\mathbf{0 . 0 8 7 0 9}$ \\
S 1 & $\mathbf{0 . 4 2 2 4 1}$ & $\mathbf{0 . 2 8 6 4 6}$ & $\mathbf{0 . 1 5 0 7 7}$ & $\mathbf{0 . 0 8 1 1 1}$ & $\mathbf{0 . 0 2 0 2 2}$ & $\mathbf{0 . 0 0 2 1 6}$ & $\mathbf{0 . 0 0 0 0 2}$ & $\mathbf{0 . 0 0 0 0 0 0 7}$ \\
\hline
\end{tabular}


Fig. 12 and Fig. 13 respectively is spectrum diagram of IMF1 and IMF2. According to the two images can be sure rolling bearing fault happened, but fault feature information is not obvious. The characteristic frequency $f_{o}$ of bearing fault in condition of experiment can be obtained by special formula [15], and $f_{o}=97.946 \mathrm{~Hz}$.

In order to accurately extract fault feature information, the envelopment analysis of IMF2 and IMF1 are made, and their envelope spectrum diagrams are Fig. 14 and Fig. 15 respectively. From the graphs can easily see that there exists $98.2 \mathrm{~Hz}, 196.4 \mathrm{~Hz}, 294.6 \mathrm{~Hz}, 392.8 \mathrm{~Hz}$ frequency components and so on, which are very close to 1, 2, 3, 4times frequency calculated with theory, and explain bearing fault happened. For comparative effectiveness of the method, the envelope analysis of IMF which is closer to original signal is made. As shown in Fig. 16 and Fig. 17, IMF6 and IMF7 are respectively the envelope spectrum diagrams. Though these can find from the 1,2, 3, 4times frequency, but the fault feature information is not obvious and it is difficult to accurately extract fault feature frequency.

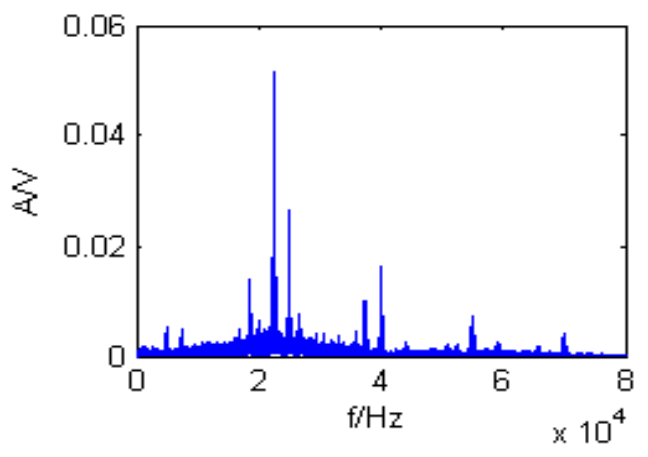

Fig. 12. The spectrum diagram of IMF1

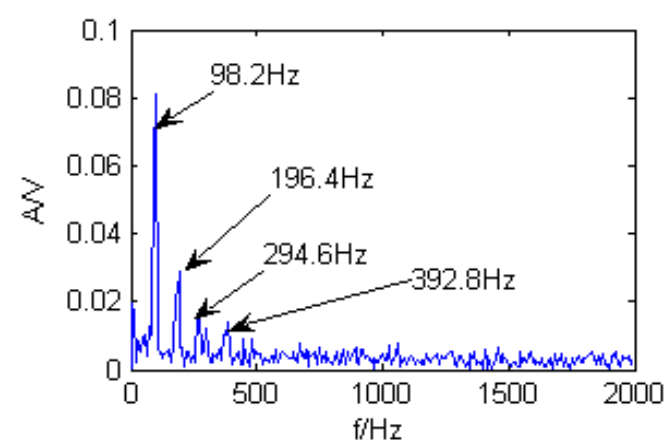

Fig. 14. The envelope spectrum diagram of IMF1

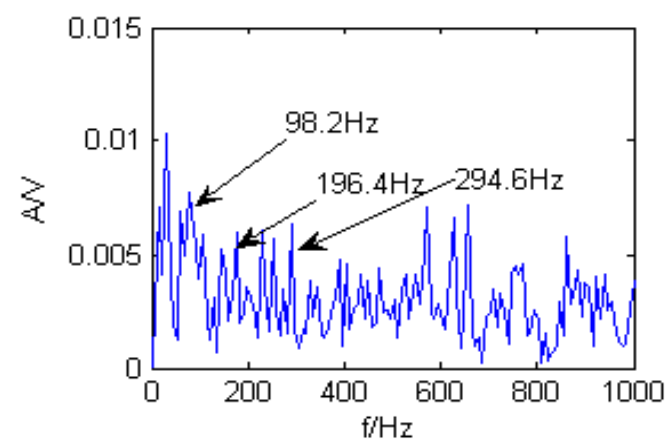

Fig.16.The envelope spectrum diagram of IMF6

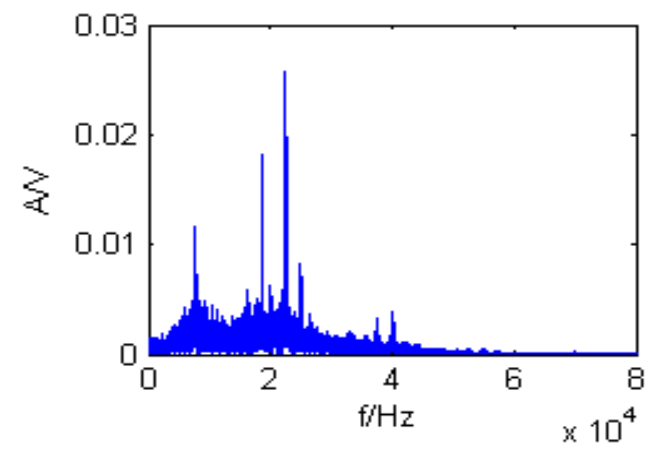

Fig. 13. The spectrum diagram of IMF2

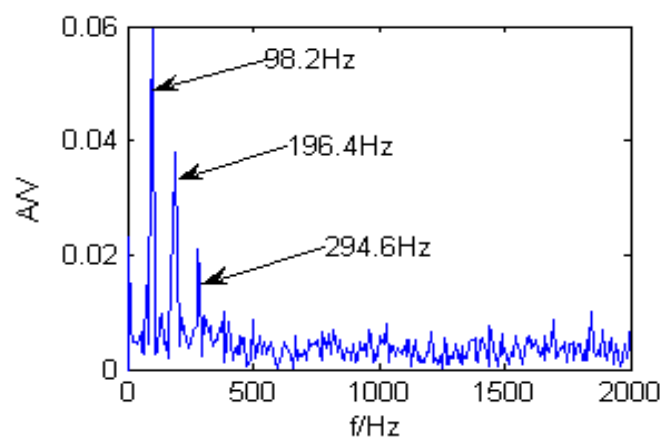

Fig.15.The envelope spectrum diagram of IMF2

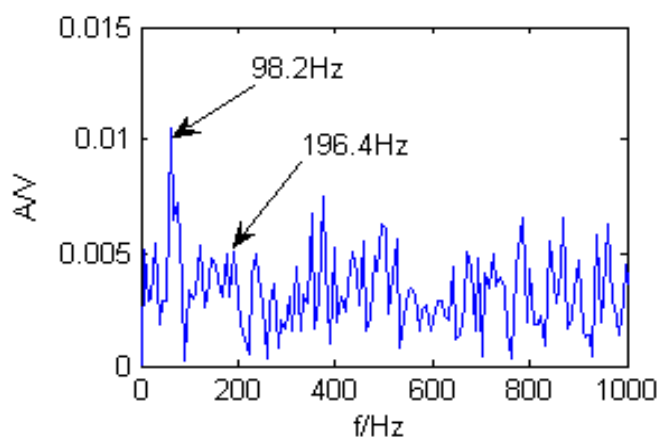

Fig.17.The envelope spectrum diagram of IMF7 


\section{Summary}

The lifting wavelet and EMD are effective way to suitable for analyzing non- stationary and nonlinear signal. But purring with any one of these methods was difficult to accurately extract useful components from the early weak fault AE signal. The method that combining with lifting wavelet transform, EMD, related function, spectrum analysis and envelopment analysis, not only can overcome the problem of EMD endpoint and a good elimination of noise, but also effectively and accurately extract fault characteristic signal, which provides an effective method for the diagnosis of early AE weak fault.

\section{Acknowledgement}

Supported by Hunan Provincial Innovation Foundation for Postgraduate, Aid Program for Science and Technology Innovative Research Team in Higher Educational Institutions of Hunan Province, Science and Technology of Hunan Province Key Project (2010FJ2012), and Natural Science Foundation of China (51075140), are gratefully acknowledged.

\section{References}

[1] Jing Shuangxi, Li Shenghua, Hua Wei. The second generation wavelet de- noising method in the application of fault diagnosis [J]. Coal Electrical Machinery, 2007, 2:3-5.

[2] Duan Chendong, He Zhengjia. The second generation wavelet de-noising method applies in fault diagnosis system [J]. Small Miniature Computer System, 2009, 2004, 25(7): 1341-1343.

[3] Shen Xiaoan. The ultrasonic signal de-noising technology based on lifting wavelet transform [J]. Engineering Design Journal, 2009, 16(3):227-229.

[4] Liu Shuchun, Pan Ziwei, Song Miao. A new method research of the second generation wavelet in vibration signal de-noising [J]. Mechanical Transmission, 2008, 3:64-68.18-21.

[5] Sun Wensheng, Wang Fengtao, Zhang Zhixin etal.EMD noise reduction and spectrum kurtosis method in rolling bearing early fault diagnosis applications [J]. Journal of Vibration and Shock, 2010,29(3)18-21.

[6] Gao Lixin, Wu Lijuan, Zhang Jianyu. An application in gear incipient failure diagnosis based on EMD demodulation method [J]. Journal of Beijing University of Technology, 2009, 35 (7): 876-880.

[7] Long Kai, Li Gangcheng. A method of incipient fault diagnosis for rolling bearing based on hilbert transform and weibull Distribution [J]. Science technology and Engineering, 2010, 10 (17):4163-4167.

[8] Cui Lingli, Gao Lixin,Duan Haichen et al. Composite Fault Diagnosis Method. Based on Second Generation of Wavelet and EMD [J]. Journal of Vibration and Shock, 2008, 27(6):1-3.

[9] Wang Fengli, Zhao Deyou. Fault feature extraction based on lifting wavelet and local wave [J]. Chinese Journal of Scientific Instrument, 2010, 31(4):789-79 3.

[10] Sweldens W. The lifting scheme A construction of second generation wavelet constructions [J]. SIAM J.Math. Aanal. 1997, 29(2):511-546.

[11]Zhang Defeng. MATLAB Wavelet Analysis [M]. Beijing: Machinery Industry Press, 2009.

[12]Cheng Junsheng, Yu Dejie, Yang Yu. Application of EMD to local rub-impact fault diagnosis in rotor systems [J]. Journal of Vibration, Measurement and Diagnosis, 2006, 26(1):24-27.

[13]Liao Chuanjun, Luo Xiaoli, Li Xuejun. Application of wavelet packet in feature extraction of acoustic emission signal [J]. Journal of Electronic Measurement and Instrument, 2008, 22 (4): 79-85.

[14]Mittrakovic D, Grabec I Sedmak S. Simulation of AE signals and signal analysis systems [J]. Ultrasonic, 1985, 23(9): 227- 232.

[15]Mei Hongbin. The Rolling bearing of vibration detection and diagnosis [M]. Beijing: Machinery Industry Press, 1996. 